\author{
Ilenia Ruggiu \\ (professore ordinario di Diritto costituzionale nell'Università degli Studi \\ di Cagliari, Dipartimento di Giurisprudenza)
}

\title{
Reati culturalmente motivati in Brasile: il caso di stupro-non stupro presso gli indios Guaranì (con riflessioni sui matrimoni precoci Rom) *
}

\author{
Culturally motivated crimes in Brazil: the case of rape/non-rape \\ among the Guarani Indians (with remarks on Roma early marriages) *
}

\begin{abstract}
This article describes a culturally motivated crime that came to the attention of a Brazilian court: sexual intercourse with a girl under the age of fourteen in a Guaranì group, indios from the Mata Atlantica. The judge acquitted the accused taking into account the fact that in the Guaranì society a woman becomes an adult with menarche and that sexual initiation is widespread in this culture. The accused had violated the protocol of this initiation, for which he will be sanctioned according to the norms of Guaranì law. The Brazilian case allows for a series of observations on the debate on Roma early marriages that is taking place in Europe, and on the different treatment between sex acts in this context and sex acts between minors under fourteen years old and other teenagers in the majority cultures of Europe.
\end{abstract}

SOMMARIO: 1. Premessa - 2. Il caso - 3. L'iter processuale e gli argomenti. Materialità del fatto ed ermeneutica antropologica - 4. Un nuovo tassello nella cultural expertise: lo strumento della "audizione etnica" (escuta étnica) - 5. Il pluralismo giuridico dialogico e l'affidamento della sanzione alla comunità guaranì - 6. Il ruolo del giudice di fronte a pratiche culturali patriarcali - 7. Dal Brasile all'Europa: spunti di riflessione sui rapporti sessuali di infraquattordicenni nel matrimonio rom e nella cultura italiana.

\section{1 - Premessa}

Nonostante la grande diversità etnica presente in Brasile, il fattore culturale non viene in genere evocato né preso in considerazione nelle corti brasiliane. Fa eccezione, in questo panorama, una decisione del Tribunale Federale di Paranaguá1, nello Stato del Paranà, riguardante un

\footnotetext{
* Contributo sottoposto a valutazione. Article peer evaluated.

${ }^{1}$ Pubblico Ministero Federale v. Valdenei Da Silva, sentenza della Sezione giudiziaria del
} 
indigeno del gruppo guaranì accusato di violenza sessuale ai danni di una tredicenne. Il giudice ha assolto l'imputato con la motivazione che non si trattava di stupro, ma di una violazione di protocollo da sanzionare in base alle regole del diritto guaranì. Si tratta del primo riconoscimento della categoria di reati culturalmente motivati in Brasile $^{2}$ e della prima decisione che riconosce l'esistenza di un diritto tradizionale indigeno, dandogli rilevanza giuridica nel decidere un caso.

La decisione è rilevante nel dibattito sui reati culturalmente motivati in corso in Italia ${ }^{3}$ in quanto contiene interessanti riflessioni su diverse questioni: l'impatto degli elementi culturali sull'ermeneutica del fatto e sulla tipicità; la possibilità di ricorrere a sanzioni alternative interne alla minoranza culturale; i limiti a pratiche culturali di matrice patriarcale. Inoltre, la sentenza è rilevante per un particolare tipo di strumento l'audizione etnica (escuta étnica) - che è stato forgiato ad hoc per risolvere il caso. Tale strumento aggiunge un tassello alla questione della cultural

Paraná, $1^{\circ}$ Distretto federale di Paranaguá, caso $\mathrm{N}^{\mathrm{o}}$ 5002882-22.2015.4.04.7000/PR, 23 marzo 2015 (inizio procedimento); decisione del 31 agosto 2018; giudice relatore Guilherme Roman Borges. La sentenza nonché tutti i documenti di seguito citati in nota e collegati al procedimento giudiziario qui analizzato mi sono stati gentilmente forniti dal giudice Guilherme Roman Borges e sono pubblici in Brasile. Chi desiderasse leggere i documenti integrali può contattarmi all'indirizzo iruggiu@unica.it. Le traduzioni dal portoghese all'italiano in tutto il contributo sono a mia cura.

2 A oggi, la Corte suprema brasiliana si è pronunciata soltanto su "manifestazioni culturali", come le pratiche della "vaquejada", (ADI 4983/CE, Rel. Min. Marco Aurélio, sentenza 1 giugno 2016) della "farra do boi" (rodei) (RE 153.531, Min Relatore Marco Aurélio, sentenza 6 marzo 1997) e del "combattimento tra galli" (ADI 18556, Rel. Min. Celso de Mello, sentenza 26 maggio 2011), ma non ha mai introdotto nelle sue argomentazioni la categoria dei reati culturalmente motivati. La sentenza in esame, pertanto, rappresenta la prima proposta di configurazione giuridica di tale categoria nella giurisdizione brasiliana.

${ }^{3}$ Nell'ambito di tale dibattito, per la prospettiva penalistica si vedano, ex pluribus: A. BERNARDI, Il "fattore culturale" nel sistema penale, Giappichelli, Torino, 2010; C. DE MAGLIE, I reati culturalmente motivati. Ideologie e modelli penali, Edizioni ETS, Pisa, 2010; F. BASILE, Immigrazione e reati culturalmente motivati. Il diritto penale nelle società multiculturali, Giuffrè, Milano, 2010. Per la prospettiva costituzionalistica e comparata si vedano, ex pluribus: P. PAROLARI, Culture, diritto, diritti: Diversità culturale e diritti fondamentali negli stati costituzionali di diritto, Giappichelli, Torino, 2016; I. RUGGIU, Il giudice antropologo. Costituzione e tecniche di composizione dei conflitti multiculturali, FrancoAngeli, Milano, 2012, nuova edizione tradotta: Culture and the Judiciary. The Anthropologist Judge, Routledge, London, 2019; P. PANNIA, La diversità rivendicata. Giudici, diritti e culture tra Italia e Regno Unito. Uno studio comparato, CEDAM, Padova, 2021. 
expertise ossia di come apportare nel processo la conoscenza antropologica necessaria per risolvere il caso.

Le argomentazioni fornite dal Tribunale Federale di Paranaguá, pur se da contestualizzare nella specificità del Brasile, arricchiscono quella conversazione giurisprudenziale sulla cultural defense che sta avendo luogo a livello comparato tra i giudici di tutto il mondo. In particolare, anche se il caso accaduto nella foresta della Mata atlantica brasiliana, a prima vista, appare lontano dall'orizzonte europeo, le argomentazioni sviluppate possono essere confrontante con alcune decisioni sui cosiddetti matrimoni precoci rom, sui quali farò qualche osservazione a conclusione dell'analisi.

\section{2 - Il caso}

Il caso che il Tribunale federale di Paranaguá si è trovato a decidere riguardava un indios Mbyá guaranì di nome Valdenei Da Silva (Werà in lingua guaranì), di anni ventinove, accusato di stupro ai danni della figliastra e nipote (figlia biologica del fratello), J.Y. Da Silva, di anni tredici. Valdenei Da Silva si era da qualche anno trasferito dalla sua comunità di origine al villaggio di Kuaray Oguatá Porã, dove si sono svolti i fatti, per sposare Tereza Gonçalves, già consorte del fratello e madre della minore J.Y., diventando uno dei cacique (leader) del villaggio.

La denuncia della violenza proveniva da un'insegnante non guaranì, Isabel Goulart da Costa, la quale, dopo aver saputo che le assenze da scuola della giovane J.Y. erano causate da un problema sentimentale con il patrigno/zio, approfondendo, aveva scoperto che la tredicenne aveva avuto rapporti sessuali con lui.

A seguito della denuncia si apriva un procedimento penale per "stupro di persona vulnerabile" ai sensi dell'art. 217-A del codice penale brasiliano che sanziona il fatto di "avere una congiunzione carnale o compiere un altro atto libidinoso con un minore di quattordici anni" con la pena da otto a quindici anni di reclusione.

Dagli atti processuali emerge che il nucleo familiare viveva in abitazioni separate: in una risiedeva l'imputato Valdenei Da Silva, in un'altra la moglie Tereza Gonçalves con la figlia J.Y., avuta dalla precedente relazione con il fratello di Da Silva. Poco dopo il menarca di J.Y., Valdenei Da Silva aveva cessato di invitare la moglie a dormire presso la sua abitazione, invitando, viceversa, la figliastra. Da questa relazione era derivata una gravidanza che la minorenne aveva occultato alla scuola, ma che era nota sia alla famiglia che al resto della comunità. Il bambino era nato morto e d'accordo con J.Y., Tereza Gonçalves e i nonni paterni 
Juventina e Faustino Da Silva, il cadavere del bambino era stato sotterrato dallo stesso padre, Valdenei Da Silva.

Se, in un primo tempo, tutta la famiglia era restata unita nell'evitare l'intervento della giustizia brasiliana, in un secondo momento, su invito dell'insegnante, la denuncia per stupro era stata sottoscritta anche dalla minorenne e dalla madre di questa, Tereza Gonçalves.

Il pubblico ministero così formulava l'accusa:

"In giorni e orari non specificati, ma certamente occorsi tra gli anni 2010 e 2013, in continuità delittiva, nella Aldeia Kuaray Guatá Porá, in località Cerco Grande, Municipio d Guaraqueçaba, Comarca de Antonina, Paraná, l'accusato Valdenei Da Silva, avvalendosi della sua condizione di patrigno, in modo cosciente e volontario, ha praticato congiunzione carnale con la vittima [...] Dall'atto praticato conseguì una gravidanza della vittima. Il neonato è morto in circostanze non chiarite".

L'avvocato di Valdanei Da Silva, previa presentazione di una perizia antropologica e psicologica di parte, chiedeva al giudice di considerarlo non imputabile con queste motivazioni:

“ $\mathrm{E}$ risaputo, Eccellenza, che $\mathrm{i}$ silvicoli ancora non hanno comprensione accurata delle diverse e complicate sfumature della società cosiddetta civilizzata [...] Quindi, quando si tratta di silvicoli, soltanto una perizia antropologica può provarne lo sviluppo mentale incompleto: se sono pienamente acculturati; se hanno uno sviluppo mentale che consente di comprendere l'illegittimità dei loro atti e infine, se sono pienamente imputabili e in grado di comprendere la portata e il significato delle loro azioni. In questo senso, la relazione peritale antropologica prodotta, costituisce una prova sufficiente per dimostrare la non imputabilità dell'imputato [...] È evidente che l'indigeno accusato soffre di qualche disturbo mentale o psicologico e/o diversità antropologica dovuti al processo di inculturazione per il fatto di essere rimasto un indio conforme ai costumi tribali del gruppo etnico guaranì del villaggio di Kuaray Guatá Porá della terra indigena di Cerco Grande, comune di Guaraqueçaba, Paraná, dato che la sua condotta reiterata esula completamente dalla normalità di un indio acculturato ${ }^{4 \prime}$.

La difesa chiedeva, quindi, l'assoluzione e "il ricovero in un ospedale di custodia e trattamento psichiatrico o in un altro [istituto] indicato dagli specialisti antropo-psicologici".

4 RD Baldino Garcia, Advogados Associados, Risposta all'accusa OAB/PR n ${ }^{\circ} 48.978$, Curitiba, 24 agosto 2018. 
Su tali due configurazioni proposte dal pubblico ministero e dalla difesa che rappresentano, in qualche modo, il volto del diritto coloniale brasiliano rispetto ai popoli indigeni - criminalizzazione senza alcun accertamento dell'altrui visione del mondo, da un lato; uso della perizia antropologica per mostrare lo scarso sviluppo di una cultura e patologizzazione della condotta indigena, dall'altro - irrompe la decisione del giudice del Tribunale federale di Paranaguá che ribalta entrambe le letture introducendo una cultural defence sottoscritta, come vedremo, anche dal pubblico ministero che si unirà alla richiesta di assoluzione.

\section{3 - L'iter processuale e gli argomenti. Materialità del fatto ed ermeneutica antropologica}

In difesa dell'imputato interviene la Fondazione nazionale degli Indios (Fundação Nacional do Índio/FUNAI) che richiede al giudice di disporre una perizia antropologica a cura di un'esperta culturale proveniente dall'accademia ${ }^{5}$. Dalla perizia emergono vari profili culturali: innanzitutto, il sistema di parentela presso il popolo guaranì, che rende frequenti matrimoni all'interno dello stesso gruppo familiare, come in questo caso in cui Teresa Gonçalves ha sposato prima uno poi l'altro fratello Da Silva; quindi, la poliginia presente nel gruppo guaranì cui appartiene l'imputato, che era diverso rispetto a quello della moglie.

Rispetto alla parentela scrive l'antropologa:

"Tanto la supposta vittima (J.Y. Da Silva) quanto il supposto aggressore (Valdenei Da Silva/Werá) sono indigeni e parenti per consanguineità (nipote/zio) che per affinità (figliastra-patrigno), appartenendo a una tcheretarã/familia estesa Guaranì, di profilo endogamico, costituita tramite alleanze incrociate (alianças cruzadas)".

Sembra, inoltre, emergere, dalle interviste condotte dall'antropologa ai vari protagonisti del caso, che nessuno percepisca il fatto come uno stupro e che lo stesso malessere manifestato dalla tredicenne a scuola non sia dovuto tanto al trauma del rapporto sessuale, che lei stessa avrebbe voluto, ma piuttosto alla gelosia, dovuta al fatto che la giovane desiderava l'esclusività con il patrigno/zio. Si legge nella perizia antropologica:

«Il sentimento di gelosia fu menzionato sia da Faustino Da Silva

5 Ana Elisa de Castro Freitas (antropologa e docente presso 1'Universidade Federal do Paraná), Pré-Laudo Antropológico, 21 marzo 2015 (18 pagine). 
[nonno paterno di J.Y.] che da Tereza Gonçalves [madre dI J.Y.] come il motore che ha spostato il trattamento del rapporto tra Valdenei Da Silva/Werá e J.Y. Da Silva [...] dall'ambito dell'organizzazione sociale Guaranì al mondo dei non indios, culminando nella criminalizzazione di Valdenei Da Silva/Werà [...] Le dichiarazioni di J.Y. suggeriscono un'opposizione alla riproduzione della tradizione guaranì della poligamia [...] Esempi di poliginia di capi e sciamani sono frequenti nella letteratura guaranì $[. .$.$] nelle generazioni più anziane la pratica$ di questi matrimoni è ancora frequente, ma nelle giovani generazioni c'è tensione sul mantenere questa tradizione. Alcuni la considerano "obsoleta e difficile da tollerare", mentre altri la considerano "condotta sacra e prova di appartenenza al gruppo dei discendenti dei 'veri' antenati Guaranì"».

La perizia dà conto anche della storia di questo gruppo guaranì e di altre dimensioni utili a inquadrarne i valori.

Il giudice ritiene necessari ulteriori approfondimenti. È in questo contesto che elabora uno strumento, forgiato $a d$ hoc, che è unico nel suo genere nella giurisdizione brasiliana ed è qui utilizzato per la prima volta. Il nomen iuris assegnato dallo stesso giudice è escuta étnica, audizione etnica o ascolto etnico (ethnic hearing) (infra par. 4). L'audizione viene realizzata recandosi personalmente presso il gruppo anziché convocare i testimoni in tribunale. I testimoni, infatti, ad avviso del giudice, sono l'intero villaggio, dal quale sarà possibile cogliere la reale portata del comportamento di Valdenei Da Silva.

Il giudice prende contatti con la comunità guaranì e, accompagnato dall'antropologa che aveva già fornito la perizia, si reca nella foresta della Mata atlantica.

Lo svolgimento concreto dell'escuta étnica inizia a porre alcuni problemi di incomprensione culturale: pur avendo un preciso appuntamento, il giudice viene lasciato ad attendere oltre un'ora. Lui interpreta il gesto come una scortesia, ma il gruppo che lo riceve gli spiega che era in raccoglimento per poter rispondere al meglio alle sue domande ${ }^{6}$. L'antropologa conferma che lungi dall'essere un gesto irriverente, l'attesa a cui l'hanno sottoposto è un segno di rispetto.

Seduti nella piazza del villaggio, inizia una conversazione in cui il giudice formula le sue domande (infra par. 4) e interloquisce con il gruppo, restando sorpreso dal fatto che anche i bambini sono chiamati a dire la loro su diverse questioni. L'attività del giudice è quella di un vero e

\footnotetext{
${ }^{6}$ Questo fatto mi è stato rivelato dal giudice relatore Guilherme Roman Borges in una intervista a me rilasciata nel giugno 2021.
} 
proprio antropologo impegnato in un lavoro di campo.

Incrociando i dati raccolti da lui stesso con quelli forniti dall'antropologa nella perizia culturale già presentata, la sentenza rifletterà ampi contenuti della cultura guaranì che vanno dalla cosmovisione degli indios, alle regole della parentela, alla religione, ai riti di accesso all'età adulta, al loro sistema giuridico. La sentenza, infatti, cerca di ricostruire tutto il sistema culturale per leggere il comportamento.

Dall'escuta ètnica emerge che il principio della buona vita (Bem Vivir) governa l'intero assetto di scelte dei guaranì, anche per quanto concerne il risanamento di trasgressioni compiute dai membri del gruppo, e che Valdenei Da Silva ha violato tale principio. Tuttavia, altrettanto chiaramente, emerge che il rapporto sessuale intercorso tra l'imputato e la minorenne non è percepito come uno stupro.

In primo luogo, esso è, per i guaranì, una relazione consenziente tra adulti. Infatti, la giovane ha già avuto il menarca. Questo è il momento fisico che, nella tribù guaranì, segna l'ingresso nell'età adulta. L'imputato Da Silva, dunque, non aveva davanti a sé una minorenne, ma una donna nel pieno possesso della sua autonomia sessuale. Si legge nella sentenza che presso il gruppo in esame:

«la divisione tra l'infanzia e l'età adulta non è scandito dal criterio dell'età che informa la capacità nel sistema giuridico comune (criterio utilizzato anche per valutare la presunzione di violenza nei reati sessuali) ma dall'avvento delle mestruazioni. È stato notato, a questo proposito, che la "vittima" aveva già le mestruazioni quando si sono verificati i fatti. Questo fatto è stato confermato durante l'audizione etnica, che ha consentito di acquisire ulteriori informazioni: dopo il menarca, la giovane trascorre un mese di isolamento, dormendo in un letto sospeso senza toccare il pavimento e mangiando cibo equilibrato nel sale etc., e si realizza tutto un procedimento che segna la sua maturazione guaranì come donna, d'ora in poi adatta per il sesso, il matrimonio e la riproduzione».

In secondo luogo, il giudice rileva che le visioni del sesso presenti nella cultura in esame sono diverse da quelle egemoniche per cui la stessa categoria di delitti sessuali va riletta con le lenti culturali guaranì. La sessualità, infatti, è percepita come qualcosa di naturale e vi si accede attraverso rituali di iniziazione sessuale portati avanti da persone più grandi, in genere parenti, nei confronti dei giovani. Si legge nella sentenza:

«Non si può dimenticare che il sesso praticato nel mondo guaranì presenta, secondo l'antropologa Ana Freitas, tratti molto diversi dal mondo "occidentale" (parola con cui il capo di Araçaí [sentito durante l'escuta ètnica] si riferisce alla cultura 
egemonica), senza questioni tipiche della cultura bianca, quali il pudore, il tabù, la privacy come imperativo, l'omosessualità come patologia [...] Poiché la sessualità è vissuta in modo diverso, è doveroso non guardare ai "reati sessuali" negli stessi termini della cultura "standard". Alla già citata diversa maturazione sessuale, che allontana eventuali prospettive di vulnerabilità come da noi intesa, si aggiungono i rapporti familiari con famiglie allargate diverse [...] il fatto che l'iniziazione sessuale è spesso data dai membri della famiglia e, sebbene suoni estraneo alla cultura dominante, da uomini più anziani nella comunità $[\ldots]$ il fatto che il matrimonio [...] è precoce e può verificarsi già a dieci, undici anni, come osservato da questo stesso magistrato [durante l'escuta ètnica]. [Per tutti questi motivi] la comprensione di "stupro" non può mai essere pensata in termini culturali egemonici».

In tale contesto, il malessere di J.Y. viene ricondotto non al fatto che la giovane abbia vissuto il rapporto come una violenza, ma al fatto che l'azione di Da Silva si sia svolta al di fuori delle prescrizioni richieste dal principio del Bem Vivir che regola anche le iniziazioni sessuali. Infatti, l'imputato ha omesso due importanti passaggi di un protocollo, fatto che rende la sua azione senz'altro biasimevole. Un primo passaggio del protocollo consiste nello spiegare alla figliastra/nipote che lui l'avrebbe iniziata alla sessualità e nell'offrirle dei doni, ritualizzando il rapporto sessuale, anziché iniziarlo, pur presente il consenso di lei, al di fuori del rito; un secondo passaggio consiste nel comunicare ai parenti della giovane che avrebbe voluto intraprendere una relazione con lei e iniziarla sessualmente.

Nessuno di questi adempimenti è stato posto in essere da Da Silva che, infatti, come emerge dalla escuta étnica, è considerato dal suo gruppo aver avuto un comportamento disdicevole, animalesco. Il caso si configura, dunque, come una violazione di etichetta dovuta a una caduta interiore di Da Silva, che si è allontanato dai principi del Bem Vivir. Per tale disequilibrio interiore, la sanzione prevista nel diritto guaranì è che Da Silva si sottoponga a un complesso rituale di purificazione sciamanico, sotto la guida degli anziani del villaggio, previa separazione dalla figliastra e dalla moglie.

Si ricostruisce, dunque, che la stessa vittima si percepiva tale per ragioni diverse da quelle ravvisate dal diritto ufficiale e che il ricorso alla giustizia ufficiale, sia da parte della minorenne che della madre, è stato determinato da un intrecciarsi di ragioni dovute in parte alla gelosia, in 
parte alla violazione di protocollo subita, ma non all'aver vissuto l'evento come uno stupro tanto che la stessa madre di J.Y. che aveva sporto denuncia, considera il carcere una sanzione eccessiva.

Alla luce di questi dati, il giudice dispone l'assoluzione "perché secondo la visione del mondo giuridico guaranì ciò che è accaduto è stata una illegittima violazione di protocollo, piuttosto che un crimine". Il giudice precisa che l'imputato non andrà esente da sanzione, ma affida questa al gruppo guaranì che si è impegnato nella sua rieducazione. Anche il pubblico ministero si unisce alla richiesta di assoluzione con «l'argomento centrale della non colpevolezza dell'imputato in quanto "errore culturalmente condizionato"».

In una sessione della sentenza intitolata "Imputazione: la sussunzione della condotta dell'autore nell' „esperienza giuridica" Guaranì e non all'articolo 217-A del codice penale», il giudice dichiara di voler utilizzare l'art. 383 del codice di procedura penale brasiliano - che così dispone:

"Art. 383. Il giudice, senza modificare la descrizione del fatto contenuta nella denuncia o nel reclamo, può attribuirgli una diversa definizione giuridica, anche se, di conseguenza, deve applicare una sanzione più grave".

Attraverso tale norma, il giudice compie una sussunzione decolonizzata:

«Ritengo necessario fare una sussunzione incrociata [...] Al contrario di come alcuni penalisti collocano la questione, nel rispetto delle posizioni contrapposte, non ritengo trattarsi di un problema di antigiuridicità o di colpevolezza [...] Penso [che ci troviamo] nel campo della "tipicità" stessa, ma di una "tipicità" [che va] letta alla luce di una "svolta decoloniale", e pertanto una "tipicità incrociata", poiché si sta analizzando un fatto attraverso un "tipo" forgiato in un'altra cultura [...] Forse si potrebbe parlare di una sorta di "atipicità incrociata o etnica"».

In questa svolta decoloniale promossa dal Tribunale di Paranaguà, il principio iura novit curia si estende a includere anche il diritto indigeno.

Come rilevato dallo stesso giudice nella decisione, in Brasile ancora manca una dottrina della Corte suprema sui reati culturalmente motivati, quindi si dovrà attendere per capire se la linea adottata dal Tribunale di Paranaguà si consoliderà. La decisione non è stata impugnata, quindi, in attesa di futuri casi, essa segna il primo riconoscimento di pluralismo giuridico a favore del diritto guaranì. 


\section{4 - Un nuovo tassello nella cultural expertise: lo strumento della "audizione etnica" (escuta étnica)}

Lo strumento della escuta étnica creato dal Tribunale Federale di Paranaguá per raggiungere la decisione in esame aggiunge un nuovo tassello alle varie tipologie di cultural expertise ${ }^{7}$ fino a oggi codificate perizia dell'antropologo, informazioni immesse da un membro qualificato della minoranza, test culturali - in quanto vede lo stesso giudice divenire artefice di una sorta di sua propria perizia culturale, forgiata e condotta da lui medesimo, con la presenza di un'antropologa in funzione di mediatrice culturale, per rispondere alle questioni che emergono nel caso concreto.

Nella definizione di Livia Holden, la cultural expertise è quella

«speciale conoscenza che consente a studiosi socio-giuridici, antropologi o, più in generale, mediatori culturali, i cosiddetti "cultural brokers", di individuare e descrivere fatti rilevanti alla luce del particolare background dei ricorrenti, delle parti in causa o della persona o delle persone accusate e, in alcuni casi, della/e vittima/e»8.

La Holden propende per una definizione ampia di cultural expertise ${ }^{9}$, non limitata alle perizie che sono in grado di fornire gli specialisti accademici o gli antropologi, ma aperta a includere tutti quegli strumenti che possano apportare al processo la conoscenza antropologica: sicuramente, dunque, le informazioni fornite dai mediatori culturali/cultural brokers, così come i test culturali di creazione giurisprudenziale o dottrinale sono dalla Holden considerati forme di cultural expertise $e^{10}$.

Anche l'escuta étnica usata nella decisione in esame può considerarsi una forma di cultural expertise di creazione giurisprudenziale, predisposta su misura per un singolo caso, ma potenzialmente esportabile anche per la risoluzione di altri e utilizzabile come ideal-tipo che ciascun giudice può adattare alle esigenze del caso che sta analizzando.

7 Sulle forme assunte dalla cultural expertise in America latina si veda da ultimo L. RODRIGUEZ (a cura di), Culture as Judicial Evidence. Expert Testimony in Latin America, University of Cincinnati Press, Cincinnati, 2020.

8 L. HOLDEN (a cura di), Cultural Expertise and Litigation: Patterns, Conflicts, Narratives, New York-London, Routledge, 2011, p. 2.

$9 \mathrm{Si}$ veda anche L. HOLDEN, Beyond anthropological expert witnessing: toward an integrated definition of cultural expertise, in Cultural Expertise and Socio-Legal Studies: Special Issue, Series Studies in Law, Politics, and Society, vol. 78, 2019, pp. 181-204.

$10 \mathrm{Si}$ veda il numero speciale della rivista Laws dedicato alle diverse forme di cultural expertise in questa definizione ampia, L. HOLDEN (a cura di), Cultural Expertise: An Emergent Concept and Evolving Practices, Special Issue, in Laws, n. 8, 2019 (open source). 
Lo strumento utilizzato in questo caso concreto consiste in quaranta domande previamente scritte dal giudice e da lui poste personalmente ai membri della comunità in un dialogo aperto e collettivo che, come visto, si è svolto - secondo il metodo antropologico - presso il gruppo, non in tribunale.

Nella sentenza, il giudice spiega come è arrivato a elaborare tale strumento. Il riferimento è ai test culturali già proposti in dottrina o usati nella giurisprudenza nord-americana. Si citano, nella sentenza, i test culturali suggeriti da Alison Dundes Renteln ${ }^{11}$ e Ilenia Ruggiu ${ }^{12}$. Il giudice, tuttavia, riconosce che le domande suggerite in questi test non sono sufficienti a risolvere in via definitiva il suo caso $^{13}$, e ha bisogno di ulteriori domande, che predispone attraverso un'ordinanza ${ }^{14}$.

Tra queste quaranta domande, ne figurano alcune generali, volte a ricostruire il sistema giuridico della comunità guaranì e il suo senso di giustizia, e altre più focalizzate sul fatto concreto. Per l'innovatività dello strumento, ritengo utile riportarlo per intero nelle sue quaranta domande:

«1) Quali elementi/argomentazioni/ragionamenti definiscono qualcosa come esemplare nella comunità?

2) Quali elementi/argomentazioni/ragionamenti definiscono qualcosa come riprovevole nella comunità?

3) Che cosa considerate come norma/regola/principio nel vostro gruppo?

4) Quando pensate a ciò che è possibile, proibito o consentito fare, pensate a che cosa? Si tratta di ciò che si aspetta la comunità, la famiglia, la natura o/e la religione?

5) Quello che considerate proibito/consentito ecc. è qualcosa di stabile (visto sempre allo stesso modo), se no, da cosa dipende la variazione nel tempo: dall'orientamento religioso? Dalla leadership della tribù? Dalle persone coinvolte nelle controversie?

6) I divieti/permessi vengono trasmessi di generazione in generazione o vengono ricreati ogni volta?

11 A. DUNDES RENTELN, The Cultural Defense, Oxford University Press, Oxford, 2004, p. 207.

12 I. RUGGIU, Culture and the Judiciary. The Anthropologist Judge, Routledge, London 2019, Cap. 4.

13 "Dopo aver risposto alle domande dei test culturali suggeriti dalla Renteln e dalla Ruggiu, mi sono reso conto che ancora non avevo tutte le informazioni e ho creato l'escuta étnica", dichiarazione rilasciata dal giudice relatore Guilherme Roman Borges nel webinar, Culture and the Judiciary, Plurilegal series, 24 marzo 2021, disponibile su youtube.

${ }^{14}$ Despacho/decisão del 30 maggio 2018. 
7) I divieti/permessi dipendono da chi ha il potere di orientamento religioso o tribale?

8) Questioni di potere fisico, spirituale, "economico", sociale, politico, ecc. condizionano la definizione di che cosa è permesso/vietato?

9) Le gerarchie familiari condizionano tali permessi/divieti?

10) Le linee guida religiose definiscono divieti/permessi? Fino a che punto e in quali termini?

11) Diverse tribù, diverse etnie, diverse regioni geografiche presentano variazioni nelle loro percezioni di cosa è permesso/vietato?

12) Esempi individuali contribuiscono alla definizione di che cosa è permesso/vietato? In breve, esempi di ciò che è giusto/sbagliato sono esaltati allo scopo di incoraggiare la comunità ad agire all'interno di ciò che è permesso/vietato?

13) Essendo [la vostra comunità] politeista, in che modo e quali istituzioni intervengono nella definizione di cosa è permesso/vietato?

14) Che cosa si intende per giusto? È qualcosa di rigido, fisso, precostituito (sempre o qualche volta)? Esiste un'idea di giustizia?

15) C'è differenza tra ciò che è permesso/proibito e ciò che è giusto o sono sempre equivalenti?

16) [Il vostro diritto] È tutto orale o c'è qualche forma di registrazione scritta?

17) Essendo orale, come si trasmette la tradizione di ciò che è permesso/proibito?

18) Quali tipi di interferenza ci sono nella definizione di ciò che è giusto? Congiunture politiche interne, fattori esterni?

19) Ci sono interferenze intertribali nella definizione di ciò che è giusto e di ciò che è permesso/proibito?

20) La costruzione di ciò che è giusto, ciò che è permesso/proibito è individuale, avviene da parte di un'autorità comunitaria/religiosa, è un processo storico-generazionale, collettivo?

21) In che modo le pratiche considerate inappropriate/ingiuste sono viste dalla comunità?

22) C'è una chiara definizione previa di chi svolge le funzioni di dire che cosa è giusto/sbagliato, permesso/vietato, giusto/sleale?

23) Da dove proviene la determinazione di ciò che è permesso o vietato: tradizione, autorità comunitarie, autorità religiose?

24) Quando arriva un nuovo soggetto nella tribù, come si adatta ai divieti/permessi? C'è qualche forma di insegnamento o impara dall'esperienza?

25) Chi decide su questo punto è la stessa "autorità" che risolve eventuali violazioni [da parte del nuovo venuto]? 
26) C'è un modo per fare appello esterno alla tribù [al sistema di giustizia ufficiale brasiliano] per definire questioni di giusto/ingiusto, vietato/consentito?

27) Ci sono distinzioni di grado di atti compiuti e considerati impropri?

28) Quali sono le forme di ricorso/denuncia [contro comportamenti proibiti]? Sono forme individuali o collettive?

29) Il ricorso/denuncia è fisso o flessibile? Cioè, varia con il tempo, il contesto, le persone coinvolte?

30) Il ricorso/denuncia segue qualche rito specifico? Quali sono le forme delle procedure interne? Dipendono dalla natura dell'atto compiuto?

31) La posizione di cacique [capo] interferisce in modo sfavorevole o favorevole nella decisione sul ricorso/denuncia quando si compie un atto contrario alla cultura della tribù?

32) Che tipo di punizione subiscono gli atti sessuali come quello per cui il signor Valdenei Da Silva/Werá è perseguito?

33) C'è una distinzione in termini di pratiche proibite/consentite più o meno gravi e disapprovate dalla comunità? Esiste la figura di reato?

34) C'è una differenza di severità/punizione perché un atto sessuale è compiuto contro un bambino?

35) Quali sono i danni interni che si generano nella comunità quando si compie un atto del genere?

36) La figura femminile come vittima di un atto sessuale è qualcosa che assume un peso diverso all'interno del comunità indigena?

37) Esistono usanze che comportano punizioni?

38) Le sanzioni sono soggette a un tipo di controllo/vigilanza interna? Nella cura di quale autorità si trova la persona punita?

39) Come si conclude l'erogazione della pena? C'è qualche atto formale che ne marchi la conclusione innanzi alla tribù? C'è qualche forma di comunicazione con la tribù di origine e le persone sanzionate?

40) Qual è la vostra posizione sul fatto che gli atti commessi dalle popolazioni indigene siano giudicati dallo Stato?»

L'obiettivo di questo strumento è così illustrato dall'antropologa che accompagna il giudice:

"La pratica dell'ascolto etnico (escuta étnica) consiste nell'instaurare una comunità argomentativa dialogica tra Stato e Popoli indigeni, che ha luogo nello spazio vitale dell'interlocutore/comunità indigena interessata, e che mira ad accedere agli elementi culturali che organizzano la sfera etica e morale della comunità [...] È molto importante creare le condizioni che permettano la comprensione, la 
riflessione e la parola e, in questo senso, la procedura richiede un atteggiamento rispettoso al fine di garantire il giusto spazio-tempo per la comprensione e l'elaborazione del discorso e il suo ascolto. Pause, silenzi, intervalli e discorsi costituiscono la durata e la temporalità dell'ascolto etnico. In questi termini, la metodologia è eminentemente dialogica. Il magistrato, dott. Guilherme Roman Borges, pone domande aperte sui grandi temi della vita sociale, come la condotta esemplare, i regimi deliberativi (problematizzando nelle sue domande temi quali, tra gli altri, equità, uguaglianza, rappresentatività, genere, età adulta, responsabilità, diritti e doveri, modalità di correzione, sanzioni)"15.

La decisione in esame inaugura, in qualche modo, un nuovo approccio all'acquisizione di conoscenza antropologica rendendola plurale e accentuando il ruolo del giudice: da un lato, c'è la classica perizia antropologica scritta, quindi le classiche testimonianze dibattimentali di soggetti vicini alla vittima o all'imputato, infine - elemento di novità - c'è il lavoro di campo condotto dallo stesso giudice che utilizza, come esperti culturali, il villaggio, andando a intervistare in loco molti più membri del gruppo di quanti acconsentirebbe una normale testimonianza dibattimentale. Il risultato è una sentenza in cui il giudice cita alternativamente l'antropologa Ana Freitas, il cacique [capo] Laercio e gli altri membri del gruppo quali fonti di conoscenza culturale.

Questa pluralità di voci consente un equilibrio tra informazioni fornite da un soggetto esterno al gruppo e imparziale (l'antropologa) e la visione del gruppo che il giudice ha modo di acquisire direttamente, senza mediazioni, facendosi lui stesso antropologo. In questa attività, il giudice opera in collaborazione con l'antropologa che, come lui stesso dichiara, partecipa alla escuta étnica "al fine di evitare di perpetrare offese culturali agli indigeni da parte del potere giudiziario"16. In queste parole, si ravvisa la fiducia del giudice nei riguardi dell'antropologia come scienza più avanti rispetto a quella giuridica nella comprensione delle differenze culturali e capace di mediare eventuali interpretazioni erronee dei dati raccolti in cui il giudice potrebbe incorrere. Questo sodalizio, questo binomio giurista-antropologo nella stessa raccolta dei dati utili a valutare

15 Notas de Campo - Escuta Étnica na comunidade Mbyá Guarani do Tekoá Kuaray Oguatá Porã/Cerco Grande Guaraqueçaba, 11/08/2018, Profa. Dra. Ana Elisa de Castro Freitas Perita Antropóloga Laboratório de Interculturalidade e Diversidade Universidade Federal do Paraná.

16 È quanto scrive il giudice nell'ordinanza in cui predispone l'escuta etnica, Despacho/decisão del 30 maggio 2018. 
l'operabilità di una cultural defense sancisce un nuovo tassello nel dialogo tra diritto e antropologia: non più due momenti separati, da un lato la perizia scritta e dall'altro il giudice che la valuta, ma un lavoro comune nella comprensione delle ragioni culturali sottese al comportamento. Lo strumento e i suoi esiti ai fini della decisione sfatano anche quell'iniziale scetticismo nei confronti della possibilità dei giudici di farsi antropologi per via della incommensurabilità culturale di certi comportamenti. ${ }^{17}$

La sentenza testimonia la necessità di strumenti ad hoc per approcciare una disputa multiculturale e può essere un esempio per gli altri giudici, a livello comparato, che volessero elaborare propri schemi motivazionali e domande da rivolgere agli imputati di reati culturalmente motivati. L'escuta étnica arricchisce il dibattito sulle tecniche e gli strumenti (test culturali, handbook sulle pratiche culturali, COI/Country of origin information, database di perizie culturali, etc.) che il giudice antropologo può porre nella sua cassetta degli attrezzi per lavorare alle sfide del multiculturalismo.

\section{5 - Il pluralismo giuridico dialogico e l'affidamento della sanzione alla comunità guaranì}

Oltre che per l'accuratezza con cui la cultural defense è motivata, la decisione in esame è rilevante perché il giudice riconosce il pluralismo giuridico inerente al Brasile e tra diritto statale e diritto indigeno opta per l'applicazione di quest'ultimo.

Un'apposita sezione della sentenza è intitolata "Per un pluralismo effettivo: pluralismo giuridico dialogico" (sec. 2.2.a). In essa, il giudice osserva che il rinnegare il modo in cui i guaranì risolverebbero il caso sarebbe un etnogiuridicidio, ossia una forma di uccisione della folk law guaranì:

"continua ad esistere una pratica tragica di oscuramento di esperienze giuridiche diverse, che forse si può definire un

${ }^{17}$ Si veda quanto scriveva A.J. CONNOLLY, Cultural Difference on Trial. The Nature and Limits of Judicial Understanding, Routledge, London and New York, 2010, p. 2: “Legal agents do not possess and are unable to adequately acquire some - perhaps, even any - of the concepts which inform the thought and practice of the members of those groups". A distanza di vent'anni da questa constatazione di incommensurabilità culturale, mi pare si possa dire che la figura del giudice antropologo, ossia del giudice che si confronta con la categoria cultura, con strumenti giuridici sempre più sofisticati, quali l'escuta étnica, riveli una crescente capacità giudiziale di maneggiare il concetto di cultura. 


\author{
"etnogiuridicidio" operato dall'egemonia violenta dell'ordinamento \\ giuridico statale».
}

Nel contempo, il giudice osserva che l'approccio volto a riconoscere esperienze giuridiche diverse non deve risolversi [in una]

"semplice" accettazione della giurisdizione indigena» ma piuttosto deve promuovere un "dialogo tra razionalità giuridiche, [che] consente l'integrazione della visione del mondo giuridica (jusmundividência) indigena nella comprensione giuridica (juscompreensão) dello Stato" evitando una sorta di "tribalizzazione" degli ordinamenti giuridici, fenomeno comune nei paesi latinoamericani di matrice spagnola comunemente noto sotto il nome di "folklorismo giuridico" (Magdalena Gómez). Ritengo che l'accettazione della giurisdizione indigena non porti [...] ad un pluralismo effettivo [... piuttosto] il pluralismo giuridico è efficace quando la multinormatività si trova di fronte a un dialogo (ecco perché "pluralismo dialogico"), con reciproche concessioni, reciproche modifiche normative, compenetrazioni di razionalità, accettazione di somiglianze e differenze, cuciture pragmatiche [...] ed autentico rispetto etico e non solo giuridico. Chissà che questo non sia un modo per realizzare il progetto di emancipazione tanto necessario per gli indigeni Brasiliani (Déborah Duprat). Riconoscere che la condotta dell'imputato deve essere letta dalla Giustizia federale (leggi: legge statale) alla luce dell' ",esperienza giuridica guaranì", in quanto è un presunto "reato culturalmente motivato" (qui visto come il comportamento di un soggetto appartenente ad un gruppo etnico minoritario considerato reato dalla cultura giuridica dominante, ma accettato come normale o approvato dalla cultura del gruppo a cui appartiene [...]), a mio avviso, implica un vero e proprio diritto di partecipazione delle popolazioni indigene alla costruzione dei loro diritti innanzi allo Stato e non solo il rispetto di una sfera isolata nel quadro costituzionale. Ritengo che riconoscere l'isolamento giurisdizionale e giuridico non sia molto lontano, in prospettiva epistemologica, dal totale assimilazionismo che ancora contraddistingue la storia delle relazioni tra indigeni e stato brasiliano».

Questo approccio è sancito in via generale. Nel caso concreto, il rapporto tra diritto statale e diritto indigeno vede una netta prevalenza del secondo, che è quello che viene applicato. Da Silva ha sbagliato e verrà sanzionato, ma secondo le regole guaranì. Il giudice riporta nella sentenza quanto emerso dall'escuta étnica:

«L'esperienza giuridica guaranì prevede fondamentalmente le seguenti punizioni: conversazione diretta e intimidatoria dei Cheramoi 
(sciamani) quando ascoltano le divinità; lavoro di comunità variabile (da dieci, venti o trenta giorni) nella semina, nella costruzione e nell'alimentazione collettiva (sebbene alcune tribù non lo facciano, come manifestato dal Cacique Laercio); e lo spostamento in un'altra tribù sotto gli auspici di un altro capo (mai il semplice bando), il cui tempo non risulta essere oltre cinque anni. I guaranì non stabiliscono mai l'esilio puro, perché comprendono il legame con la terra che gli indigeni hanno, e perché sono, in larga misura, imparentati. Le tribù Guaranì sono sempre costituite da piccole famiglie (Cerco Grande ha attualmente cinque famiglie e Araçaí una trentina). Inoltre non istituiscono prigioni (sebbene alcuni gruppi etnici prevedono "gabbie", come emerge dall'escuta étnica) e non la pena di morte, perché incompatibile con Namanduí, o principio creativo, anteriore al tutto».

Attingendo all'universo giuridico guaranì, il giudice trova nella sanzione con cui il villaggio rieducherà $\mathrm{Da}$ Silva uno strumento più efficace rispetto allo sradicamento e conseguente indebolimento della comunità che deriverebbe dalla sanzione di diritto ufficiale.

\section{6 - Il ruolo del giudice di fronte a pratiche culturali patriarcali}

Un topos argomentativo che si è consolidato, a livello comparato, tra giudici che si trovano di fronte a dispute multiculturali è quello di interrogarsi sull'impatto che la pratica culturale può avere sui diritti delle donne. Sulla base di un indirizzo ${ }^{18}$ che trova la sua massima espressione nel diritto internazionale - dove ci si è maggiormente impegnati per la deculturalizzazione di molti comportamenti sostenendo che non di cultura si trattava, ma di pratiche patriarcali - molti cultural crimes sono divenuti, progressivamente, gender crimes, sottraendosi, spesso per espressa scelta legislativa o per scelta giurisprudenziale, a ogni possibilità di cultural defense.

Il lettore che ha avuto la pazienza di seguirmi fin qui potrebbe avere il dubbio che la decisione in esame, nella sua sensibilità verso le problematiche dei popoli indigeni, abiliti, in qualche modo, la

${ }_{18}$ Com'è noto, tale questione fu posta a tema per la prima volta dalla dottrina da $\mathbf{S}$. OKIN, Is multiculturalism bad for women?, in J. COHEN, M. TOWARD, M.C. NUSSBAUM (a cura di), Is multiculturalism bad for women? Susan Moller Okin with respondents, Princeton University Press, 1999, poi in Diritti delle donne e multiculturalismo, traduzione italiana a cura di a cura di A. BESUSSI e A. FACCHI, Raffaello Cortina, Milano, 2007, p. 9 ss. 
perpetuazione del patriarcato in questo gruppo: se riti di iniziazione sessuale e forme di poliginia, coinvolgenti anche infra-quattordicenni, non sono sanzionati dall'ordinamento in nome della cultura non si sta in qualche modo impedendo la modifica di comportamenti potenzialmente lesivi dei diritti delle donne e delle bambine?

Se lo è chiesto anche il Tribunale di Paranaguà, che non lascia inevasa la questione. Il giudice, infatti, ammette che alcune delle pratiche culturali alla base del comportamento del reo hanno risvolti patriarcali e crea un'apposita sezione della sentenza dedicata a "Rispetto dei diritti delle donne e delle bambine" in cui ragiona dell'approccio del giudice:

"Riconoscere l'ordine di valori che informa la cultura indigena, impedendo la lettura del fatto commesso da Werá/Valdenei Da Silva come un crimine, non significa cancellare del tutto il comportamento e ignorare il ruolo della legge statale nella promozione dei diritti delle donne e ragazze, compreso il suo ruolo nel promuovere la loro protezione dentro la cultura indigena. È noto che le donne e le ragazze sono segmenti vulnerabili in entrambe le culture, sia egemone che indigena. Quindi, sorge la domanda: la legge statale ha la funzione/capacità, attraverso l'applicazione del diritto penale, di proteggere e promuovere i diritti delle donne nella cultura indigena? [...] Soprattutto in questo caso, l'applicazione del diritto penale poco o nulla apporterebbe alla promozione dei diritti delle donne, essendo [il carcere] una punizione non riconosciuta [dalle stesse donne guaranì] e improduttiva. Il riconoscimento, invece, che si tratta di una condotta inappropriata, violatrice di diritti, che deve essere sanzionata all'interno dei riti indigeni, contribuisce a rafforzare il cambiamento all'interno della cultura indigena [contro comportamenti scorretti] [...] Evidentemente va ribadito che nessuna cultura può violare i diritti umani e la protezione delle ragazze e delle donne indigene. Naturalmente, anche la violenza domestica intraetnica è inaccettabile sotto ogni condizione, invece, il cambiamento culturale a favore dei diritti delle donne deve essere operato dall'interno dell'esperienza giuridica indigena, data la sua complessità, la sua peculiarità, e non imposto dalla legge statale punitiva, a pena di arbitrarietà e violenza".

Poiché durante la escuta étnica era emerso che le donne guaranì non consideravano Da Silva uno stupratore e la stessa Tereza Gonçalves, madre della vittima, considerava la prigione una sanzione eccessiva, il giudice ritiene che il soddisfacimento dei diritti delle donne sia meglio soddisfatto con la sanzione del diritto guaranì. Il comportamento scorretto dell'imputato verso la giovane non è passato inosservato dalla comunità la 
quale ha già predisposto meccanismi per sanare la vulnerabilità della vittima e curare l'imputato.

La sentenza aiuta a riflettere sui rapporti tra potere giudiziario e legislativo rispetto al ruolo dell'uno e dell'altro nel risolvere problemi strutturali come quello della discriminazione di genere. Il compito principale di rimuovere oppressioni strutturali attraverso politiche come l'istruzione, il sollievo dalla povertà, la ridistribuzione della ricchezza etc. spetta al potere legislativo, non alla magistratura. Ad esempio, il giudice non può ordinare la costruzione di una scuola o di quelle politiche utili a creare le situazioni che consentirebbero ai guaranì scelte riproduttive a un'età più avanzata. In qualche modo, il giudice arriva tardi. È vero che le sentenze, pronunciate "in nome del popolo", esprimono anch'esse la sovranità popolare e possono essere degli importanti strumenti che smantellano situazioni di oppressione strutturale di soggetti vulnerabili, tuttavia, in questo caso, bene ha fatto il giudice a evitare una "sentenza manifesto" sui diritti delle donne perché essa sarebbe stata avulsa dalla realtà concreta in esame e avrebbe obnubilato l'altra oppressione coloniale - che il gruppo vive.

C'è la tragedia del popolo guaranì dietro la decisione del Tribunale di Paranaguà, un popolo decimato dall'alcool, dai suicidi, dalla povertà anche per le ardue condizioni di vita in cui il governo brasiliano li ha costretti privandoli di terre e risorse.

Il fatto che il Tribunale non nasconda la violenza che lo stato brasiliano perpetua ai danni di questo popolo, mostra come il giudice che ha a che fare con questioni multiculturali spesso tenga in conto dello stato di vulnerabilità e di discriminazione in cui versa il gruppo: «non si può ignorare che la criminalizzazione indigena è soltanto una delle forme di selezione penale operata dalle istituzioni sociali e statali di repressione», scrive il giudice.

Evitando la criminalizzazione di Valdenei Da Silva tramite l'argomento culturale, il giudice sta proteggendo un gruppo vulnerabile, cercando una soluzione di equità che da un lato soddisfi le esigenze di riparazione della vittima e le esigenze della comunità di non vedere espunto un altro dei suoi membri. La questione dei diritti delle minoranze dentro le minoranze trova sollievo con i meccanismi sanzionatori previsti dallo stesso gruppo.

\section{7 - Dal Brasile all'Europa: spunti di riflessione sui rapporti sessuali di infra-quattordicenni nel matrimonio rom e nella cultura italiana}


Spostandoci dal Brasile dei Guaranì a vicende più vicine, la decisione in esame può fornire, mutatis mutandis, spunti su una delle questioni al tavolo dei giudici degli stati europei: quella dei cosiddetti "matrimoni precoci" Rom ${ }^{19}$.

Com'è noto, il diritto multiculturale va sviluppandosi attraverso un approccio topico ${ }^{20}$ : è, cioè, il frutto di quella che potremmo chiamare una "conversazione giurisprudenziale" che caso dopo caso, sentenza dopo sentenza va consolidando o scartando argomenti, fino ad arrivare a quelli più persuasivi. Nell'ottica di tale conversazione giurisprudenziale, che va ricostruita a livello comparato per meglio cogliere i punti dove si va affermando un consensus e avere lo spettro progressivo delle argomentazioni, la decisione che proviene dalla giurisdizione brasiliana merita di essere confrontata con alcune recenti decisioni pronunciate in Europa.

Il problema di rapporti sessuali con infra-quattordicenni come pratica culturale si è presentato nelle aule dei tribunali di alcuni stati europei in casi coinvolgenti imputati Rom. Presso alcune comunità Rom è, infatti, ammesso il matrimonio anche con infra-quattordicenni e i rapporti sessuali praticati nell'ambito di tali unioni hanno dato luogo a denunce per atti sessuali con minore.

L'universo culturale Guaranì è diverso da quello Rom e per distanza geografica e storia $\mathrm{i}$ due gruppi potrebbero sembrare incommensurabili. Tuttavia, almeno nella pratica culturale in esame - i rapporti sessuali di infra-quattordicenni - vi sono alcune analogie che giustificano tale comparazione: in entrambi gli universi culturali il raggiungimento dell'età legittima per avere rapporti sessuali è fissata non dalla legge, ma dall'evento naturale della maturità sessuale, per cui con il raggiungimento del menarca la donna è considerata adulta; in entrambe le culture manca la concezione del minore legale come soggetto vulnerabile, in quanto questo è considerato adulto molto prima di quanto avvenga, attualmente, nella cultura maggioritaria ${ }^{21}$; in entrambi i gruppi le scelte

19 Personalmente ritengo che il nome da dare a questa pratica sia quello neutro di "matrimoni di infra-quattordicenni". Infatti, matrimoni precoci, matrimoni di minori, matrimoni tra bambini sono tutti nomi che riflettono il punto di vista della maggioranza, mentre le persone coinvolte non sono considerate minorenni né bambine presso i Rom dove, analogamente a quanto accade tra i Guaranì, è il menarca e la maturazione sessuale a sancire l'ingresso nell'età adulta.

20 T. VIEHWEG, Topica e giurisprudenza, traduzione italiana a cura di G. CRIFÒ, Giuffrè, Milano, 1962 (Topik und Jurisprudenz, C.H. Beck, München, 1953).

${ }^{21}$ Come rilevato dal classico lavoro dello storico Philippe Ariès questa era una realtà 
sessuali/matrimoniali precoci sono ricollegate a uno stile di vita che si svolge al di fuori della cultura maggioritaria e che vede la primazia di altri valori: un'attitudine molto libera e naturale verso il sesso e l'idea che sia opportuno che un individuo più adulto possa iniziare il giovane alla sessualità per i guaranì; un alto valore assegnato alla famiglia e alla procreazione, piuttosto che all'autorealizzazione attraverso il lavoro per i rom. Infine, ciò che accomuna i due gruppi, aldilà dei profili culturali, è la situazione di marginalizzazione in cui vivono rispetto alla società maggioritaria e il fatto che i due universi normativi seguiti dall'una e dall'altra minoranza portino spesso a situazioni in cui il diritto penale della maggioranza si trova a indebolire una comunità già ferita. Di questo, soprattutto, ha tenuto conto il giudice brasiliano scegliendo di decidere il caso secondo le regole guaranì ed evitando così che il diritto penale statale perpetuasse l'ennesima espulsione di un soggetto da una comunità vulnerabile.

Per queste ragioni, può essere utile una comparazione tra il caso guaranì descritto in questo lavoro e il modo in cui i matrimoni precoci Rom sono regolati in Europa.

Due decisioni esemplificano l'approccio rispetto a questi casi.

La prima è del Tribunale di Sassari del 27 novembre 2012 che ha condannato a un anno di reclusione un giovane Rom di ventitré anni, convivente con una infra-quattordicenne, sempre Rom, per atti sessuali con minorenne ai sensi dell'art. 609 quater c.p. italiano. La difesa dell'imputato era impostata su due argomenti: la cultural defense, secondo cui i due erano sposati secondo il rito Rom, e l'assenza di uno stato di soggezione, in quanto la relazione era paritaria. Il Tribunale di Sassari riconosceva che i due avevano una relazione $\mathrm{d}^{\prime}$ amore ${ }^{22}$ e teneva in conto che il divario di età tra i due soggetti non era così elevato, ma su questi elementi faceva prevalere la ratio della norma, volta a punire qualsiasi rapporto con infra-quattordicenni se il divario di età nella coppia eccede i

anche in Europa fino al XIX secolo quando l'idea dell'infanzia si è consolidata: P. ARIÈS, Padri e figli nell'Europa medievale e moderna, Laterza, Roma-Bari, 2006 traduzione italiana a cura di M. GARIN (L'enfant et la vie familiale sous l'Ancien Régime, Libraire Plon, Paris 1960).

22 I due erano sposati secondo il rito rom, che Letizia Mancini ha opportunamente chiamato "matrimonio invisibile" per evidenziare come tali unioni matrimoniali siano prive di qualsiasi rilevanza giuridica nell'ordinamento italiano: L. MANCINI, Il matrimonio invisibile e $i$ diritti del minore. Riflessioni a partire da una recente sentenza, in $\mathrm{L}$. LUIS EFRÉN RìOS VEGA, I. RUGGIU, I. SPIGNO (a cura di), Justice and culture. Theory and practice concerning the use of culture in courtrooms, Editoriale Scientifica, Napoli, 2020, p. 213 ss. 
tre anni (attualmente elevato a quattro). La decisione è stata confermata dalla Corte d'appello di Cagliari, sezione distaccata di Sassari, con sentenza del 9 marzo del 2016 e dalla Corte di Cassazione con sentenza n. 53135 del 201723. In tale decisione, la Corte di Cassazione argomenta che: "nella cultura rom è ammesso il matrimonio anche con minori di quattordici anni, spesso avuncolato, zio/zia e nipote", ma la "giurisprudenza ha sempre ritenuto la sua non validità nell'ordinamento italiano" 24 , e afferma che la convivenza more uxorio di un maggiorenne con una minore di anni sedici viene sanzionata a prescindere dalla realizzazione di condotte correttive $\mathrm{o}$ induttive $\mathrm{o}$ dall'abuso di una posizione dominante $\mathrm{o}$ autorevole.

La seconda decisione exemplum di un'analoga tendenza giurisprudenziale è della Corte d'appello di Brasov ${ }^{25}$ (Romania) del 22 settembre 2021 che, ai sensi dell'art. 220 c.p. romeno, ha condannato per atti sessuali con minore, anche in questo caso, a un anno di reclusione il marito (con rito Rom)/convivente di una infra-quattordicenne Rom, che era rimasta incinta. Nonostante i due vivessero insieme in un rapporto sereno, il giudice ha respinto la cultural defense (precedentemente accolta dal Tribunale di primo grado di Brasov, sentenza 22 aprile 2021, che aveva assolto l'imputato) con questi argomenti: una persona minorenne è facilmente influenzabile per cui il suo consenso non è attendibile; il corpo di una infra-quattordicenne non è sufficientemente sviluppato per avere rapporti sessuali che possono comportare una gravidanza; la giovane madre è totalmente dipendente dal compagno; sussiste uno stato di pericolo in quanto la madre rinuncia alla scuola e la sua capacità di crescere un bambino è ridotta poiché lei stessa è una bambina; il comportamento produce effetti a lungo termine sulla madre (abbandono scolastico con conseguenti poche opportunità di lavoro e impossibilità di

23 Corte di Cassazione italiana, sez. III penale, n. 53135, 22 novembre 2017.

${ }^{24}$ Corte di Cassazione italiana, sez. III penale, n. 53135, 22 novembre 2017, considerato in diritto. Qui la Corte cita una sua precedente decisione, sez. 6-2, ordinanza n. 22305, 27 settembre 2013, RV 627927-01, con cui aveva "cassato il decreto con il quale era stata accolta la impugnazione del decreto di espulsione di un extracomunitario coniugato con rito 'rom', e convivente, con una donna in stato di gravidanza, non potendosi attribuire, in questo o in quell'ordinamento statale, rilevanza giuridica al matrimonio 'rom', neppure come unione di fatto regolata".

25 Sono grata al magistrato Silviu Cătălin Enache per avere illustrato questo caso durante il corso di formazione Cultural diversity in the courtroom: judges in Europe facing new challenges organizzato dall'European judicial training network EJTN e dal Max Planck Institute for Social Anthropology, Barcellona (18 e 19 novembre 2021). 
accedere a salari alti) e sul bambino (il livello di istruzione della madre influenzerà lo sviluppo del bambino).

Confrontando le decisioni dei giudici italiano e romeno con quella del giudice brasiliano si possono rilevare differenze e analogie.

Una prima differenza verte sul peso conferito al fattore culturale nel compiere l'ermeneutica del fatto. Il giudice brasiliano ha concluso per la non sussunzione nella fattispecie argomentando che non si trattava di "stupro di persona vulnerabile", ma di "una violazione di protocollo in un atto sessuale tra adulti". In questa decisione la cultural defense è apertamente adottata e incide sulla configurazione del fatto tipico, al punto che quel comportamento non è più un crimine.

La decisione brasiliana è sfidante perché propone un fatto materiale - un uomo adulto ha avuto rapporti sessuali con una tredicenne - la cui disciplina giuridica è sancita - ai sensi del codice penale federale brasiliano, un rapporto sessuale con una tredicenne è una violenza sessuale - ma l'irrompere del fattore culturale porta il giudice a compiere un'ermeneutica del fatto diversa che lo sottrae alla configurazione di crimine di stupro di persona vulnerabile (art. 217-A c.p. brasiliano) per farlo ridiventare quello che è nell'universo guaranì: una violazione di protocollo in un atto di iniziazione sessuale tra adulti.

I giudici italiano e rumeno, viceversa, non si discostano dal dato letterale del codice penale, che intende proteggere il minore sanzionando il rapporto sessuale realizzato con un infra-quattordicenne anche se dentro un'unione volontaria e sancita da un matrimonio con rito rom. Tuttavia - e qui emergono elementi di analogia - si può ritenere che sia il giudice italiano che quello rumeno abbiano ricorso a una sorta di cripto-cultural defense. Infatti, l'argomento culturale, da entrambi formalmente respinto, in qualche modo, ritorna nella scelta di una pena lieve rispetto ai minimi e massimi edittali: un anno di reclusione.

In effetti, il codice penale italiano, a differenza di quello brasiliano, consente di distinguere il rapporto sessuale avuto da una persona infraquattordicenne: se c'è il consenso, si applica l'art. 609 quater c.p., mentre se tale consenso manca o è stato ottenuto con l'inganno, si rientra nella fattispecie dell'art. 609 bis c.p., che contempla il fatto più grave della violenza sessuale. Similmente dispone il codice penale rumeno. La possibilità di accedere a una fattispecie meno grave rispetto a quella brasiliana ha probabilmente reso meno complesso trovare un contemperamento tra le esigenze repressive del sistema e il riconoscimento, sia pure non esplicito, dell'elemento culturale per i giudici italiano e rumeno. 
Proseguendo nel raffronto tra i casi guaranì e rom, un'altra differenza rispetto alle argomentazioni del Tribunale di Paranaguà è che $i$ tribunali italiani e rumeno non fanno cenno alla situazione di marginalizzazione in cui si trovano le comunità Rom né per spiegare il contesto dei matrimoni precoci né per valutare l'impatto delle pene, sia pur lievi, sulla comunità 26 . Il giudice brasiliano pone al centro della sua decisione la necessità di un diritto decolonizzato, capace di rimediare a ingiustizie del passato e, in questo contesto, forza alcuni istituti giuridici. Vista la analoga condizione dei Rom, un riferimento al contesto di marginalizzazione in cui i matrimoni precoci si inseriscono avrebbe aiutato a contestualizzare meglio la pratica.

Nel caso della Romania, ad esempio, risalendo alle radici storiche del matrimonio precoce rom, emerge che esso è nato non come una pratica culturale condivisa e dotata di valore per il gruppo rom, bensì come una pratica di protezione da un abuso sistemico. Infatti, in tale paese, per secoli i Rom sono stati schiavi e il padrone soleva abusare delle giovani schiave vergini. La pratica di far sposare le ragazze appena maturavano sessualmente serviva a renderle meno appetibili, proprio in quanto non più vergini, e le famiglie vi ricorrevano come una strategia per evitare lo stupro da parte del padrone ${ }^{27}$. Questa pratica culturale, oggi tanto aborrita dalla maggioranza rumena, affonda le radici in un'oppressione, che, in qualche modo, la maggioranza riperpetua condannando i rom per il fatto di praticarla.

Nel caso dell'Italia, come evidenziato in dottrina ${ }^{28}$, il matrimonio precoce si configura spesso come una soluzione per un gruppo che, a causa della sua emarginazione, non ha alternative di vita paragonabili alle scelte lavorative dei giovani della maggioranza: date ai rom le stesse condizioni di partenza della maggioranza e smetteranno di sposarsi precocemente, è, in sintesi, l'argomento. Anche in questo caso, la

26 Altre volte la stessa Cassazione italiana aveva rilevato l'importanza di non criminalizzare i Rom. Si veda il caso del manghel, l'accattonaggio/questua rom, Corte di Cassazione italiana, sez. V penale, n. 44516, 17 settembre 2008.

27 Sono grata all'antropologa Harika Dauth per questa informazione. Risalgono già al 1385 le prime testimonianze di rom schiavi, costretti a lavorare i campi di alcuni monasteri. La pratica della schiavitù si è protratta fino al 1855. Si veda L. PIASERE, $L a$ stirpe di Cus. Costruzioni e storie di un'alterità, Cisu editore, Roma, 2011, che inquadra la situazione dei Rom in Romania per quattrocento anni come il più grande, sistematico, controllato sistema schiavistico dell'Europa moderna.

${ }^{28}$ L. MANCINI, Il matrimonio invisibile, cit. 
criminalizzazione sembra aggravare, piuttosto che ridurre, la condizione di marginalizzazione dei rom.

Interrogarsi sull'opportunità della sanzione penale rispetto ai giovani ventenni rom nei casi esaminati, è importante anche considerando che esiste un "equivalente culturale" nella società maggioritaria, che riceve un diverso trattamento.

A causa della liberalizzazione dei costumi e dell'accorciamento dell'infanzia, anche nella cultura maggioritaria si registrano con più frequenza rapporti sessuali di infra-quattordicenni. Il codice penale italiano tiene conto di questa trasformazione: così, rispetto a un primo divieto assoluto di rapporti sessuali per gli infra-quattordicenni, il mutamento di costumi ha imposto all'attenzione del legislatore la necessità di dare rilevanza alla libertà sessuale del minore almeno di tredici anni. L'art. 609 quater, terzo comma, attualmente prevede:

"Non è punibile il minorenne che, al di fuori delle ipotesi previste nell'articolo 609 bis, compie atti sessuali con un minorenne che abbia compiuto gli anni tredici, se la differenza di età tra i soggetti non è superiore a quattro anni".

In tal modo, il codice penale italiano riflette interamente la cultura maggioritaria. La norma protegge, infatti, la pratica culturale che vede frequenti casi di fidanzatini italiani di tredici e diciassette anni che hanno rapporti sessuali, ma non la coppia Rom che si sposa dato che, generalmente, il matrimonio con il rito rom si realizza quando l'uomo è intorno ai vent'anni. L'ipotesi del più stabile progetto relazionale rom, resta fuori dall'orizzonte culturale protetto dal codice penale. Si potrebbe dire che l'impatto di un matrimonio precoce è molto maggiore di quello di una relazione sessuale precoce, per cui la diversità di regolazione è giustificata, tuttavia è difficile non trovare stridenti e, in qualche modo, viziati da un approccio monoculturale, i due diversi esiti sanzionatori.

Indubbiamente, la tematica dei rapporti sessuali di infraquattordicenni è un complesso crocevia di diritti del minore: libertà sessuale e di scelta, da un lato, e integrità psico-fisica, potenziale vulnerabilità, dall'altro, si fronteggiano rendendo la scelta ordinamentale complessa.

La decisione della giurisdizione brasiliana qui annotata, scegliendo di non ricorrere alla sanzione penale e insistendo sulla marginalizzazione di cui soffrono i guaranì, traccia una via, a mio avviso utile, per un'apertura al riconoscimento dell'argomento culturale nel contesto dei matrimoni rom, che attenui l'attuale monoculturalismo, a vantaggio di fatto dei soli rapporti sessuali fra infraquattordicenni italiani, presente 


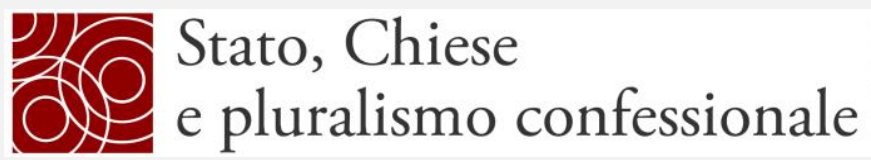

nell'art. 609 quater c.p. Un approccio caso per caso, che tenga conto della situazione di disparità e vulnerabilità del minore nel matrimonio rom $\mathrm{e}$ lasci spazio alla cultural defense se queste non si ravvisano, sarebbe forse preferibile piuttosto che una criminalizzazione tout court che aggrava la marginalizzazione dei Rom e indebolisce la comunità. 